Nig. J. Biotech. Vol. 38 (1): 179-193 (June 2021)

ISSN: 01891731

Available online at

NIGERIAN JOURNAL OF

BIOTECHNOLOGY

http://www.ajol.info/index.php/njb/index

and www.biotechsocietynigeria.org

DOI: https://dx.doi.org/10.4314/njb.v38i1.21

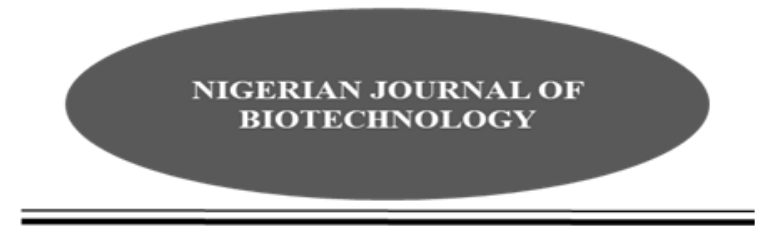

\title{
Specialized trace elements and volatile fatty acids interactions for enhanced methane production and biomethanization process stability during high organic loading rate
}

\author{
Ezebuiro, N. C. ${ }^{\text {a* }}$ and Körner I. ${ }^{\mathrm{b}}$ \\ a,bHamburg University of Technology, Eissendorfer Straße 42, D-21073 Hamburg, Germany. \\ aNational Biotechnology Development Agency, Umar Musa Yar'Adua Expressway Abuja, Nigeria.
}

\begin{abstract}
Volatile fatty acids (VFAs) and trace elements (TEs) interactions (VFAs*TEs) during biomethanization have effects that could be exploited to enhance anaerobic digestion (AD) of biomass. The goal of this study was to validate biocatalytic effects of specialized VFAs*TEs identified from a batch-derived Optimum TEs Configuration (or simply 'Optimum') on high organic loading rate (OLR) involving mixed fruit residue (MFR) fed in semicontinuous AD operation. The specialized VFAs*TEs were formulated as Variants of the Optimum and included Optimum -Cobalt (Co) for specialized VFAs*Co effects, and Optimum +Selenium (Se) for specialized VFAs*Se effects. Four duplicate AD reactors were treated with formulations reflecting the Optimum and the Variants. Each duplicate reactor was semicontinuously fed with MFR at varying OLR until instability occurred. Methane production, total volatile organic acidity (FOS) / total alkalinity (TAC) and VFAs fingerprints were measured as main responses. The results showed that reactors of the Optimum and its Variants were unstable at OLR of $8 \mathrm{~g} \mathrm{ODM} / \mathrm{L} / \mathrm{d}$, but stability was restored in the Optimum Co (FOS/TAC values of 0.6 compared to 1.51 and 1.67 for Optimum and Optimum +Se respectively). The average specific $\mathrm{CH}_{4}$ production $(\mathrm{Nm} / \mathrm{g} O D M)$ of the Optimum and its

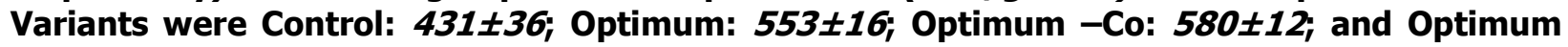
+ Se: $545 \pm 13$. Optimum -Co also had the lowest acetic acid and butyric acid accumulation, but had higher propionic acid concentration $(0.7 \mathrm{~g} / \mathrm{L})$ compared to the Optimum $(0.3 \mathrm{~g} / \mathrm{L})$ and Optimum +Se (0.4 g/L).
\end{abstract}

Keywords: Process stability, volatile fatty acids, trace elements interaction, organic loading rate, biocatalysis

*Corresponding author's email: n.c.ezebuiro@nabda.gov.ng

Introduction

Biomethanization involves biochemical processes and pathways that favor methane production during biomass degradation. Anaerobic digestion (AD) reactors are supplemented with trace elements (TEs) during biomethanization to maximize the processes of methane $\left(\mathrm{CH}_{4}\right)$ production, volatile fatty acids (VFAs) degradation and process stability (Braga et al., 2018; Mancini et al., 2018; Yazdanpanah et al., 2018). TEs improve biomethanization by enhancing the biocatalytic potentials of metalloenzymes (MEs) that are associated with $A D$, reduce digester acid accumulation and improve $\mathrm{CH}_{4}$ production (Ezebuiro and Koerner, 2017; Ezebuiro et al., 2018; Da Silva et al., 2021). Managing digester acidification is important because VFAs accumulation beyond critical levels of $200 \mathrm{mmol} / \mathrm{L}$ may lead to biomethanization failure (Zhang et al., 2010; Bardi and Aminirad, 2020). It has been reported that different configurations of TEs serve 
different $A D$ phases due to the differences in the enzymology of the pathway(s) that characterize $A D$ phases (Ezebuiro and Koerner, 2017; Ezebuiro et al., 2018).

Biochemical effects of TEs vary widely when used to enhance biomethanization and unexplainable variations due to TEs supplementation have limited the use of TEs for industrial optimization of $A D$ processes (Ariunbaatar et al., 2016; Facchin et al., 2013; Qiang et al., 2013). Research showed that while individual TEs are important (Pobeheim et al., 2011; Ünal et al., 2012; Myszograj et al., 2018), the most important effects originate from the interaction between TEs (TE*TE) and between a TE and VFAs (VFAs*TE). These interaction effects influence substrate hydrolysis, acidification, acetogenesis and methanogenesis (Ezebuiro et al., 2018; Ezebuiro and Koerner, 2017). For most biomass used as substrates in $A D$, acidification during biomethanization is related to the organic loading rate (OLR). Controlling digester acidification is important because biomethanization instability has been reported to set in with accumulation of VFAs, especially propionic acid (Zhang et al., 2010).

Earlier researches involving derivation of optimum TEs concentrations and modelling of VFAs*TEs were conducted in batch mode using VFAs mixtures as substrate (Amani et al., 2010; Ezebuiro et al., 2018). It is known that digester solids influence bioavailability of TEs (Tokalioğlu et al., 2003; Alonso et al., 2009; Maharaj et al., 2018); hence, the use of actual biomass to confirm special VFAs*TEs effects has become necessary. This not only allows for detailed studies of the special TEs interactions using industry-grade biomass fed in more practical semi-continuous mode, but also enables the variation of the OLR of the biomass to determine the constraints or limits of biocatalysis of observed VFAs*TE effects. Therefore, the objectives of the current study include to:

1. Adapt specialized VFAs*TEs observed with batch-identified Optimum TEs Configuration (OTC or 'Optimum') for high organic loading in a semicontinuous $A D$ operation using mixed fruit residue (MFR) as substrate;

2. Validate the process efficiency of the adapted TEs interactions in (1) with regards to improving process stability, methane production and VFAs fingerprints; and

3. Determine the process efficiency constraints or boundaries of the adapted TEs interactions with regards to process stability, methane production and VFAs fingerprint.

\section{Materials and Method}

This study is a follow-up to an earlier publication (Ezebuiro et al., 2018). While current method and materials are discussed in detail, published method and materials are summarized and referenced.

\section{Specialized TEs Interactions that} Influence VFAs Degradation Rate: The special interactions that influenced VFAs degradation rate were identified from the effects of an Optimum TEs Configuration derived from the published Response Surface Model (RSM) showing the synergistic and antagonistic influences of TEs on VFAs metabolism (Ezebuiro et al., 2018). The procedures for identification of an Optimum TEs Configuration (OTC) and derivation of the specialized TEs interactions, as they relate to the current study are summarized.

Identification of an Optimum TEs
Configuration: The OTC or "Optimum" (mg/L) include the $\mathrm{Ni}, \mathrm{Co}$, Se and Mo concentrations that simultaneously maximized VFAs degradation rate and $\mathrm{CH}_{4}$ production in the biomethanization of circa $120 \mathrm{mmol} / \mathrm{L}$ VFAs mixture (medium VFAs level) comprising acetic-, propionic- and butyric acids. The OTC was identified from a mesophilic batch experiment involving 24 units of 1 -litre reactors fed with VFAs mixture and supplemented with $\mathrm{Ni}, \mathrm{Co}$, Se and $\mathrm{Mo}$ in varying concentrations of low-, medium- and high levels. The supplementation matrix was designed statistically using the Custom Design Module of JMP 10. The experiment was run following the procedures recommended for the determination of methane potential of biomass (Verein Deutscher Ingenieure, 2006).

Liquid samples were taken at 3 to 4 days intervals from the reactors and analyzed for VFAs concentration and degradation rate. The data from VFAs degradation rates were further analyzed with the response surface model (RSM) to determine how $\mathrm{Ni}, \mathrm{Co}, \mathrm{Se}, \mathrm{Mo}$ and VFAs 
concentrations (factors) and their interactions influence VFAs degradation rate. To maximize the influence of the significant factors and derive the optimum factor concentration or the OTC, the desirability function proposed by Suich and Derringer, (1980) was applied to the RSM.

Derivation of Specialized TEs Interactions: The specialized TEs were derived from Figure 1 that described the behavior of VFAs degradation rate at $37^{\circ} \mathrm{C}$ as a result of $\mathrm{Ni}, \mathrm{Co}$, Se and $\mathrm{Mo}$ supplementation to VFAs concentrations between 28 and $213 \mathrm{mmol} / \mathrm{L}$ as published in Ezebuiro et al. (2018). At $a=0.05$, VFAs*TEs terms with $p$-value (Prob $>|\mathrm{t}|$ ) less than 0.05 or having negative estimate were considered specialized interactions.

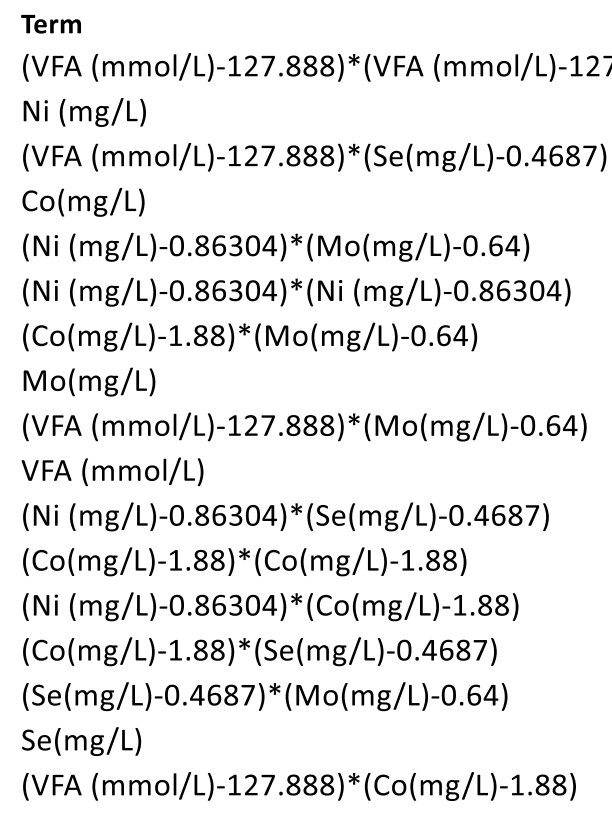

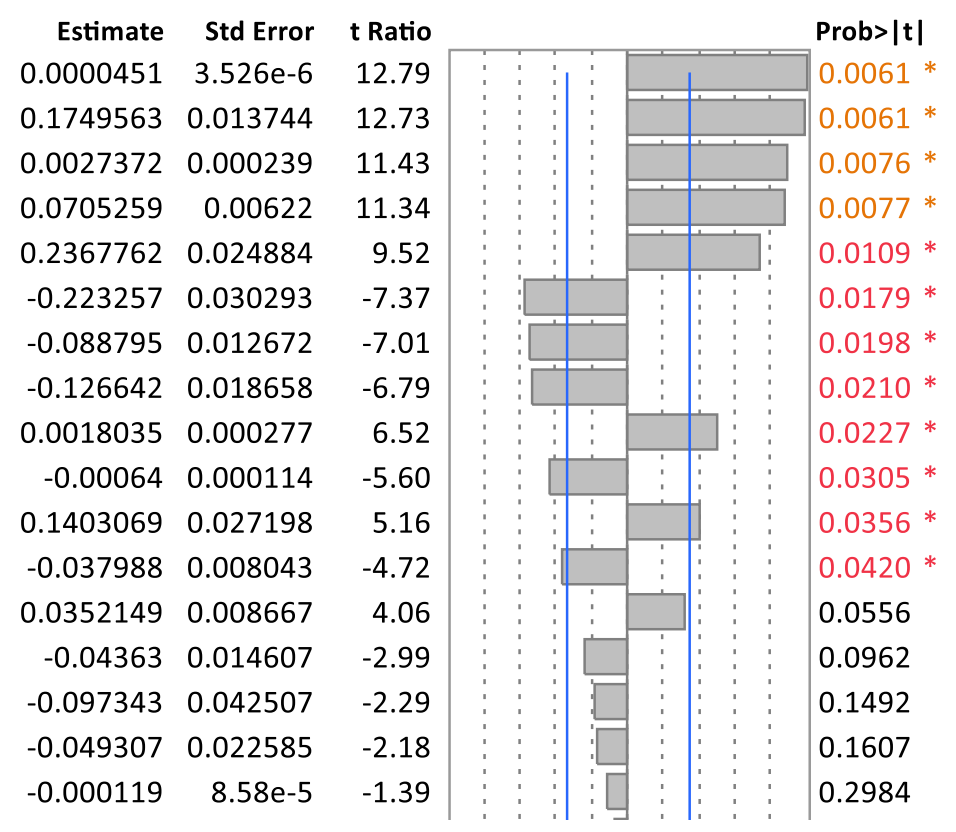

Figure 1 RSM terms (TEs and VFAs*TEs), estimates (magnitude and orientation of the terms) and significance of the terms that described the behavior of VFAs degradation rate at $37^{\circ} \mathrm{C}$ as a result of $\mathrm{Ni}$, $\mathrm{Co}$, Se and Mo supplementation to VFAs concentrations between 28 and $213 \mathrm{mmol} / \mathrm{L}$ (Adopted from Ezebuiro et al., 2018). Note: Prob > /t/ is the probability of obtaining the estimated value of the parameter if the actual parameter value is zero.

\begin{abstract}
Adaptation of Specialized TEs Interactions: The current study adapted published OTC and specialized VFA*TE effects to high organic loading rate using industry-grade biomass mixture (mixed fruit residue) as substrate. The specialized VFA*TE were constituted as alternative conditions (or variants) to which the OTC could be subjected to further enhance biomethanization. VFA*Se, VFA*Mo and VFA*Co were considered specialized interactions from Figure 1 , but VFA*Se and VFA*Co were further evaluated in the current study. Based on the orientation of the Estimates of VFA*Se and VFA*Co, the following variants of the OTC were derived:
\end{abstract}

Optimum + Se Variant: This Variant of the OTC highlights the VFAs*Se effect on process resilience during acid accumulation at reactor acidification level above $200 \mathrm{mmol} / \mathrm{L}$ VFAs. The Optimum +Se Variant contains the OTC but also an increase in Se concentration from $0.50 \mathrm{mg} / \mathrm{L}$ that is optimum for VFAs concentration $\leq 120$ $\mathrm{mmol} / \mathrm{L}$, to $1.50 \mathrm{mg} / \mathrm{L}$ that is modelled as more beneficial for VFAs levels $\geq 200 \mathrm{mmol} / \mathrm{L}$.

Optimum -Co Variant: This Variant highlights the effect of Co supplement in VFAs*Co, which suggests that Co concentration should increase above the OTC at lower OLR and vice versa (Ezebuiro et al., 2018). It was proposed to validate the assumption that a decrease in Co concentration in the OTC will confer process resilience during acid accumulation at reactor acidification level $\geq 200 \mathrm{mmol} / \mathrm{L}$ VFAs. Hence, the Optimum -Co Variant contains the OTC but 
the concentration of Co was decreased from $2.70 \mathrm{mg} / / \mathrm{L}$ that is optimum for VFAs concentration $\leq 120 \mathrm{mmol} / \mathrm{L}$ to $0.50 \mathrm{mg} / \mathrm{L}$ that

Table 1: TEs configuration of the 'Optimum' and its Variants used for reactor dosing in the semicontinuous $A D$ experiment

\begin{tabular}{lllll}
\hline $\begin{array}{l}\text { Supplementation } \\
\text { arrangements (Reactors) }\end{array}$ & \multicolumn{4}{c}{$\mathbf{~ m g / L}$} \\
\hline Control (R-1) & Co & Se & Mo \\
Optimum (OTC) (R-2) & 0.09 & 0.04 & 0.00 & 0.04 \\
Optimum -Co (R-3) & 1.46 & $\mathbf{2 . 7 0}$ & $\mathbf{0 . 4 0}$ & 0.50 \\
Optimum +Se (R-4) & 1.46 & $\mathbf{0 . 5 0}$ & 0.40 & 0.50 \\
\hline
\end{tabular}

Note: The Control TEs configuration is the TEs content and composition of the inoculum used for the current study
Validation and Determination of the Practical Constraints or the Process Efficiency Boundaries of the Specialized TES Interactions: To determine the practical biochemical constraints or boundaries of the OTC and its Variants namely Optimum + Se and Optimum -Co, $A D$ experiments were conducted in semi-continuous mode in $2000 \mathrm{~mL}$ reactors using MFR as substrate. The experimental reactors contained TEs formulations (Table 1), nutrient solution, MFR and inoculum. The experimental MFR used in this study was a mixture of different fruit residues from a fruit processing company in Hamburg. It was blended is modelled as more beneficial for VFAs concentration $\geq 200 \mathrm{mmol} / \mathrm{L}$. The OTC or Optimum and the Variants are shown in Table 1. continuous AD experiment

$\begin{array}{llll}1.46 & 2.70 & 0.40 & 0.50\end{array}$ to consistent slurry and stored at $-18^{\circ} \mathrm{C}$ until ready for use. Carbon (C) and Nitrogen (N) composition of the MFR were determined based on DIN EN 15104:2011-04 (E); and Ni, Co, Se and Mo contents of the MFR were determined as described in DIN EN 13346:2001-04. The inoculum used for the experiment was the digestate obtained from a mesophilic facility digesting maize silage composite. The TEs compositions of the inoculum were determined following the methods described for the MFR. The MFR was loaded to the mesophilic reactors in semi-continuous mode following the feeding schedule in Table 2.

Table 2: Organic loading rate of mixed fruit residue used as substrate during the mesophilic semicontinuous AD experiment

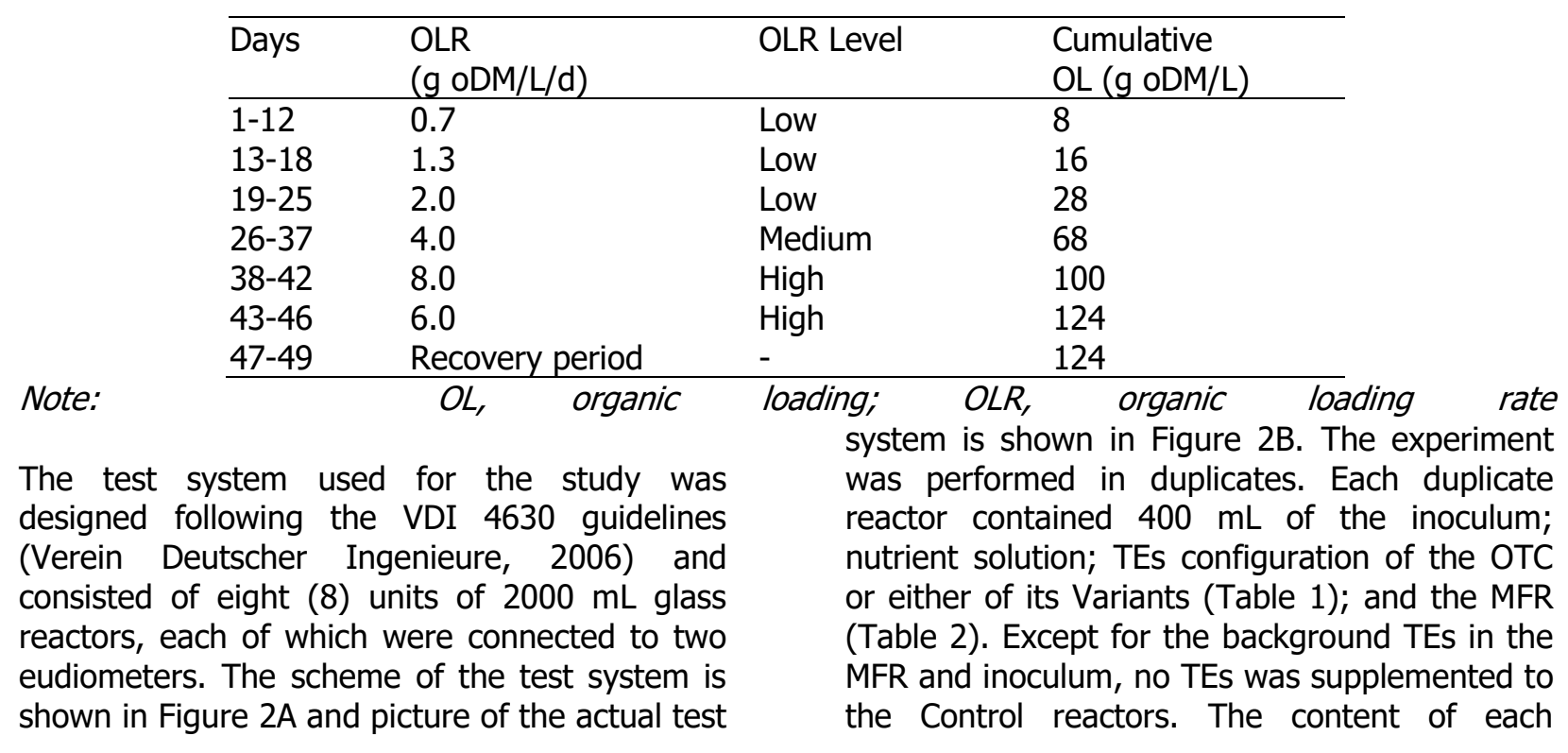


duplicate reactor was made up to $1600 \mathrm{~mL}$ with distilled water, thereby providing a headspace of $400 \mathrm{~mL}$ or $20 \%$ of the reactor volume.

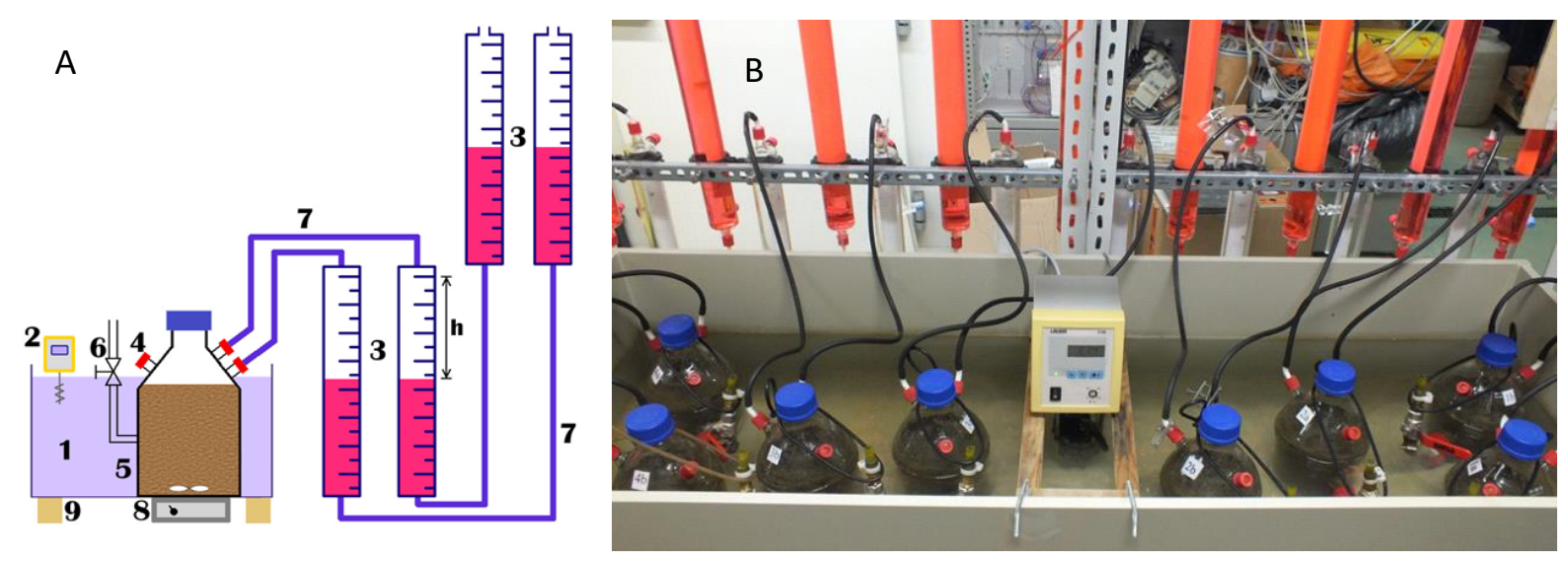

Figure 2(A) Basic scheme of the test system used for the validation investigations (1) water bath (2) thermostat (3) eudiometers (4) gas sampling port (5) glass reactors (6) valve for feeding and liquid sampling (7) reactor-eudiometer connecting tubes (8) magnetic stirrer and (9) water bath support; and (B) Actual picture of the validation test system.

The loaded reactors were placed in a water bath that was maintained at $37^{\circ} \mathrm{C}$ using a thermostat. A magnetic stirrer-bar was placed inside each reactor and eight magnetic stirrers were placed beneath the water bath such that each reactor was stirred by a magnetic stirrer through the magnetic stirrer-bar in the reactor. The loaded reactors were further flushed with dinitrogen gas for one minute to displace any oxygen (Verein Deutscher Ingenieure, 2006). During the experiments, liquid and gas samples were taken from the reactor every 3 to 4 days using a $50 \mathrm{~mL}$ syringe and biogas volume was measured within the same interval. The specific $\mathrm{CH}_{4}$ production was derived as the ratio of cumulative $\mathrm{CH}_{4}$ produced over a period to the total organic load fed to the reactor in the same period.

Table 3 summarizes the standard methods used for the analysis of the experimental parameters that include biogas volume (normalized), \% $\mathrm{CH}_{4}$, volatile organic acidity (FOS), total alkalinity (TAC), pH, and individual VFAs concentration.

Table 3: Equipment, standard methods and modification to standard methods used for sample analyses during the semi-continuous AD study

\begin{tabular}{llll}
\hline Parameter & $\begin{array}{l}\text { Sample } \\
\text { phase }\end{array}$ & $\begin{array}{l}\text { Equipment/ } \\
\text { Standard Method }\end{array}$ & $\begin{array}{l}\text { Modifications } \\
\text { to (Standard) } \\
\text { Method }\end{array}$ \\
\hline (1) Biogas volume & Gas & $\begin{array}{l}\text { VDI 2006: Eudiometer method and } \\
\text { normalization was as described } \\
\text { therein. }\end{array}$ & - \\
& & Biogas Analyzer 5000, UK & - \\
(2) $\mathrm{CH}_{4}$ content (\%) & Gas & DIN 38404 - 5 & - \\
(3) $\mathrm{pH}$ & Liquid & PRONOVA (FOS/TAC) 2000 & $15 \mathrm{~mL}$ instead \\
(4) $\mathrm{FOS}$ & Liquid & Analysentechnik GmbH \& Co. KG & of $50 \mathrm{~mL}$ \\
& & & \\
\hline
\end{tabular}




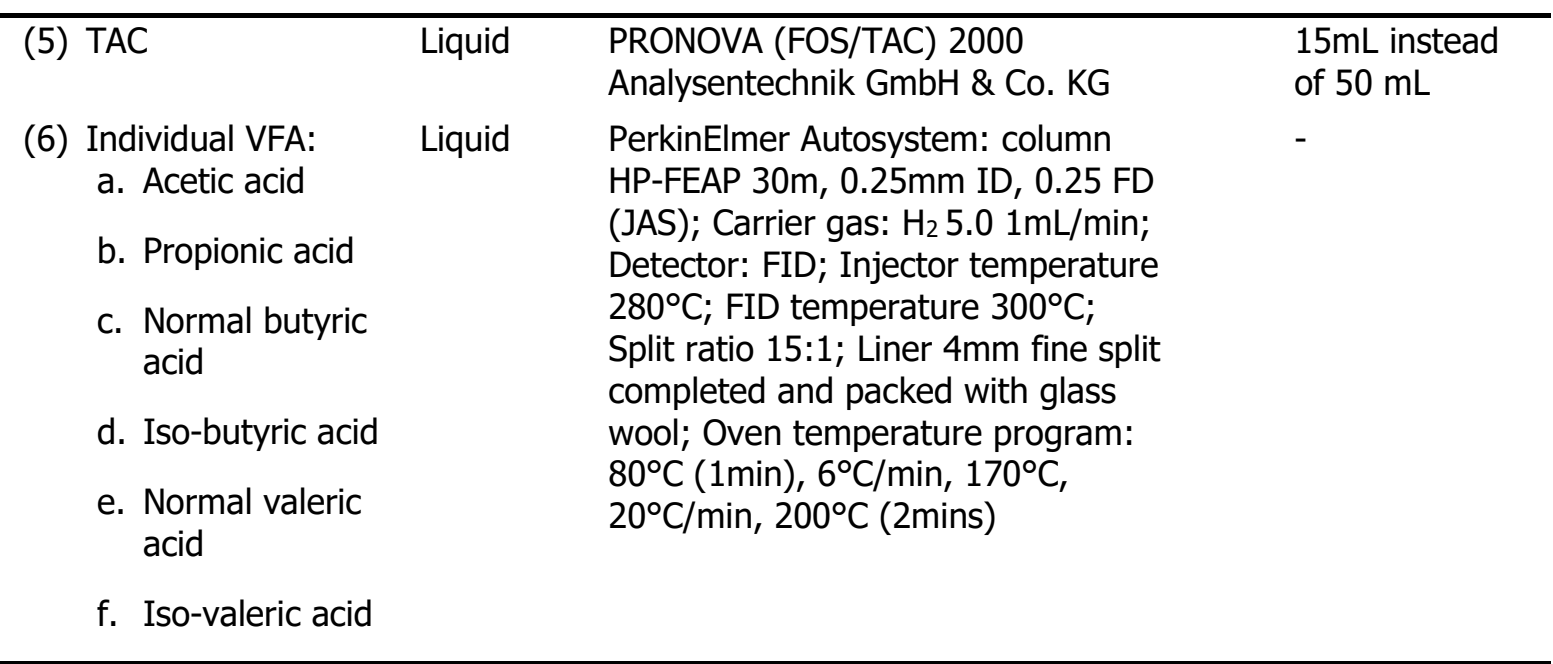

\section{Results}

Specialized TEs Interactions that Influence VFAs Degradation: From Figure 1, the significant synergistic TEs and VFAs interactions (VFAs*TE) for optimized VFAs degradation were the VFAs*Se, Ni*Mo, VFAs*Mo and $\mathrm{Ni}$ Se; and the antagonistic interaction was Co*Mo. With emphasis on the orientation of VFAs*TE (i.e., positive or negative values of Estimate), VFAs*Se, VFAs*Mo and VFAs*Ni were positive. This suggests that for optimum VFAs degradation, Se, Mo and Ni concentrations must increase beyond the OTC as VFAs levels increase in the digester. Se*Mo was negative and practically implies that the process of VFAs degradation requires either Se or Mo but not both. Hence, an increase in Se in an optimum supplementation formula intended for biocatalysis should be accompanied by a decrease in the Mo concentration. Furthermore, VFAs*Se was more positively significant for VFAs degradation (0.0076) compared to VFAs*Mo (0.227) and VFAs*Ni (0.5124). Therefore, VFAs*Se is considered a superior Variant of the OTC relative to VFAs*Mo and VFAs*Ni. The positive significance of VFAs*Se means that when VFAs concentration increases above 128 $\mathrm{mmol} / \mathrm{L}$, supplemented Se concentration should also increase above $0.5 \mathrm{mg} / \mathrm{L}$.

Regarding negative influences, VFAs*Co was the only negative VFAs*TE in the VFAs degradation model. Although statistically insignificant $(a=$ 0.05) within the limits of the studied concentrations (Table 1), VFAs*Co has an inverse relationship with VFAs concentration. Hence, VFAs*Co is considered a probable
Variant of the OTC; and Figure 1 shows that for optimum VFAs degradation, VFAs concentration greater than $128 \mathrm{mmol} / \mathrm{L}$ requires Co concentration less than $1.88 \mathrm{mg} / \mathrm{L}$.

\section{Validation of Process Efficiency and Determination of the Efficiency Constraints of the Optimum TES Configuration and its Variants}

The MFR used in this study had a dry \%DM content of $10.79 \pm 0.08$; $\mathrm{C}$ and $\mathrm{N}$ content (as $\% \mathrm{DM})$ of $45.52 \pm 0.05$ and $2.2 \pm 0.03$ respectively; and $\mathrm{C} / \mathrm{N}$ of 21 . The $\mathrm{Ni}, \mathrm{Mo}$ and $\mathrm{Co}$ contents $(\mathrm{mg} / \mathrm{kg} \mathrm{DM})$ of the MFR were $0.63 \pm 0.08, \quad 0.6 \pm 0.13$ and $0.63 \pm 0.18$ respectively. The concentrations of $\mathrm{Ni}, \mathrm{Co}$, Se and $\mathrm{Mo}$ in the experimental inoculum have been shown in Table 1.

Methane Production at OLR of $\mathbf{2 g}$ ODM/L/d: The specific $\mathrm{CH}_{4}$ production of a reactor gives indications of its biochemical efficiency at converting carbon source to $\mathrm{CH}_{4}$. Figure 3a-c shows the specific $\mathrm{CH}_{4}$ production of the reactors (Table 1 ) at the different OLR (Table 2). Figure 3a shows Specific $\mathrm{CH}_{4}$ production during the OLR of $0.7-2.0 \mathrm{~g}$ $\mathrm{oDM} / \mathrm{L} / \mathrm{d}$ in the OTC and its variants. Optimum + Se reactor showed relative weakness in specific $\mathrm{CH}_{4}$ production due to instability arising from the negative influences of higher-thanoptimum Se concentration at comparatively low OLR (0.7 to $2 \mathrm{~g}$ oDM/L/d compared to $4.0 \mathrm{~g}$ $\mathrm{oDM} / \mathrm{L} / \mathrm{d}$ in Figure $3 \mathrm{~b}$ ). At the end of $2 \mathrm{~g}$ oDM/L/d OLR, the specific $\mathrm{CH}_{4}$ ( $\mathrm{Nml} / \mathrm{g}$ oDM) produced by the reactors were: Control, 
589 $\pm 39 ;$ Optimum, 612 \pm 6 ; Optimum -Co, 635 \pm 6 ; and Optimum +Se, $552 \pm 10$.

Methane Production at OLR of $\mathbf{4 g}$ oDM/L/d: The specific $\mathrm{CH}_{4}$ production during the OLR of $4 \mathrm{~g}$ oDM/L/d is shown in Figure $3 \mathrm{~b}$. At the end of this period (Day 37), the specific $\mathrm{CH}_{4}$ production ( $\mathrm{Nml} / \mathrm{g}$ oDM) of the reactors were as follows: Control, 538 \pm 11 ; Optimum, 625 \pm 4 ; Optimum -Co, 645 \pm 10 ; and Optimum +Se, $610 \pm 5$; and these amounted to $16 \%, 20 \%$ and $13 \%$ more methanogenic activities compared to the Control reactor.
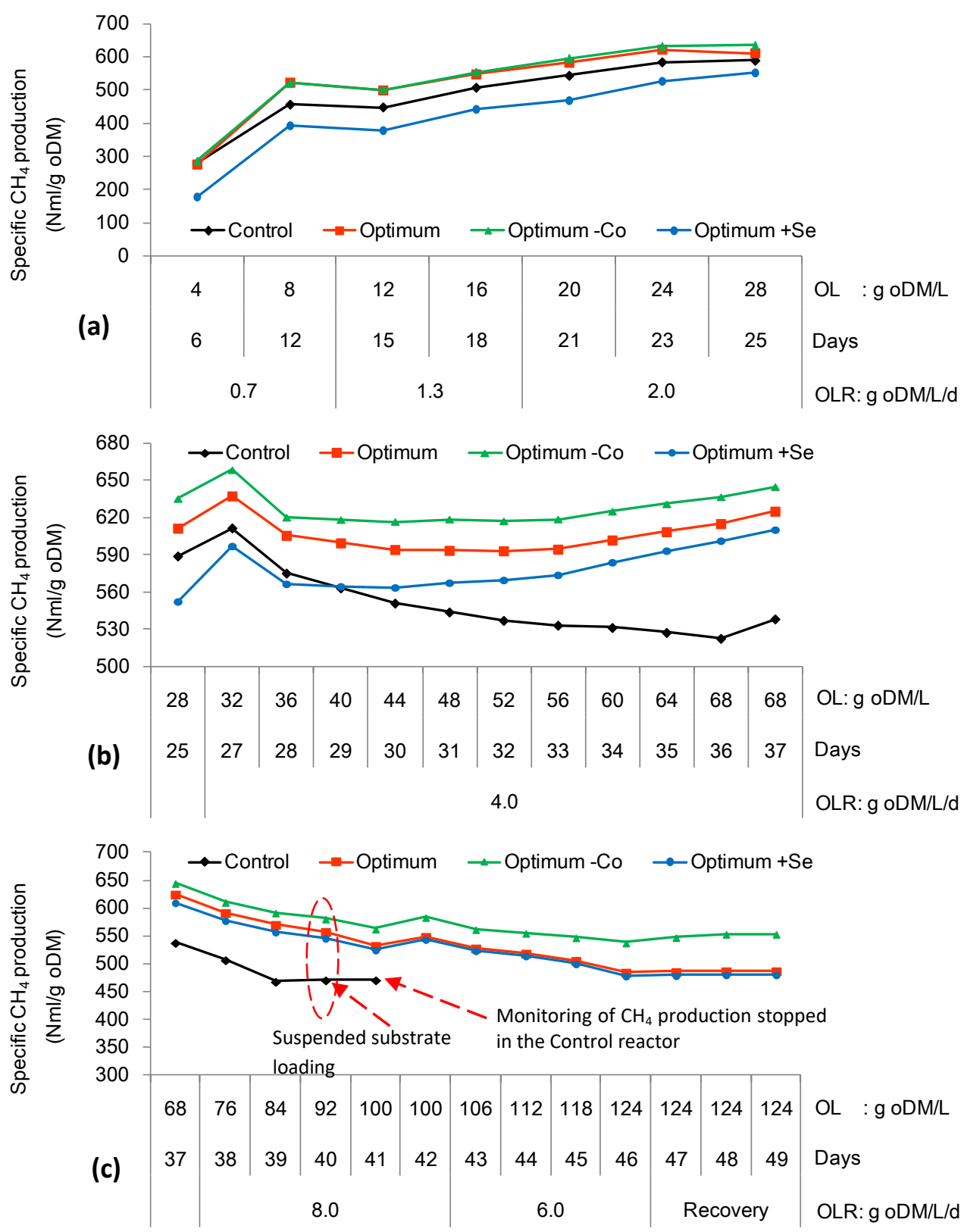

Figure 3a -c Specific $\mathrm{CH}_{4}$ production of the OTC and its Variants due to Ni, Co, Se and Mo supplementation at different OLR: (a) OLR: $2 \mathrm{~g}$ oDM/L/d (b) OLR: $4 \mathrm{~g}$ oDM/L/d (c) OLR: $8 \mathrm{~g}$ and $6 \mathrm{~g}$ $\mathrm{oDM} / \mathrm{L} / \mathrm{d}$

Methane Production at OLR of $8 g$ oDM/L/d and the Recovery Period: Figure 3c shows the specific $\mathrm{CH}_{4}$ production between the high OLR of $8 \mathrm{~g} \mathrm{oDM} / \mathrm{L} / \mathrm{d}$ and the recovery period 
when OLR was reduced to $6 \mathrm{~g}$ oDM/L/d due to VFAs accumulation in the reactors. The specific $\mathrm{CH}_{4}$ production ( $\mathrm{Nml} / \mathrm{g}$ oDM) of the Control reactor was $538 \pm 11$ at the end of the $4 \mathrm{~g}$ oDM/L/d (day 37), but decreased to $507 \pm 6$ after the first OLR of $8 \mathrm{~g}$ oDM/L/d induced reactor acidification ( $\mathrm{pH}$ of 6.2 , day 38 ). The specific $\mathrm{CH}_{4}$ production $(\mathrm{Nml} / \mathrm{g}$ oDM) of the Control reactor further decreased to $468 \pm 5$ on day 39 even though $\mathrm{pH}$ was adjusted to 7.0 on day 38 using $1 \mathrm{M} \mathrm{NaOH}$. At this time (day 39), the TEs supplemented reactors had specific $\mathrm{CH}_{4}$ production ( $\mathrm{Nml} / \mathrm{g}$ oDM) of $571 \pm 1$ (Optimum);

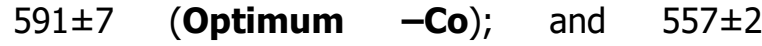
(Optimum +Se) compared to $468 \pm 5$ in the Control. This corresponded to $22 \%$ (Optimum), 26\% (Optimum -Co) and 19\% (Optimum $+\mathrm{Se}$ ) more $\mathrm{CH}_{4}$ production per day than the Control reactor. Organic loading and $\mathrm{CH}_{4}$ production measurement were suspended indefinitely on day 39 in the Control reactor due to acidification.

At the end of the OLR of $8 \mathrm{~g}$ oDM/L/d (day 42), the specific $\mathrm{CH}_{4}$ production $(\mathrm{Nml} / \mathrm{g}$ oDM) of the TEs supplemented reactors were as follows: Optimum, 549 \pm 9; Optimum -Co, 584 \pm 2 ; and
Optimum +Se, 544 \pm 4 . Instability in biomethanization due to acidification induced general decline in specific $\mathrm{CH}_{4}$ production in the TEs supplemented reactors and necessitated reducing the OLR to $6 \mathrm{~g}$ oDM/L/d on day 43 , and final suspension of digester loading on day 46 . A three-day recovery period was allowed and parameter measurements were stopped on day 49 in all the TEs supplemented reactors. The specific $\mathrm{CH}_{4}$ production ( $\mathrm{Nml} / \mathrm{g}$ oDM) of the TEs supplemented reactors at the end of the experiment on day 49 were: Optimum, $487 \pm 6$; Optimum -Co, 554 \pm 2 ; and Optimum +Se, $481 \pm 5$. The Optimum -Co reactor had $14 \%$ and $15 \%$ more specific $\mathrm{CH}_{4}$ production than the Optimum and the Optimum +Se reactors respectively.

Process Stability (Acidity/Alkalinity): The ratio of volatile organic acidity to total alkalinity (FOS/TAC) was used as indication of process stability. Figure 4 shows an overview of FOS/TAC, OLR of the reactors and the total organic load fed to the Control, Optimum, Optimum $-\mathrm{Co}$ and Optimum + Se reactors.

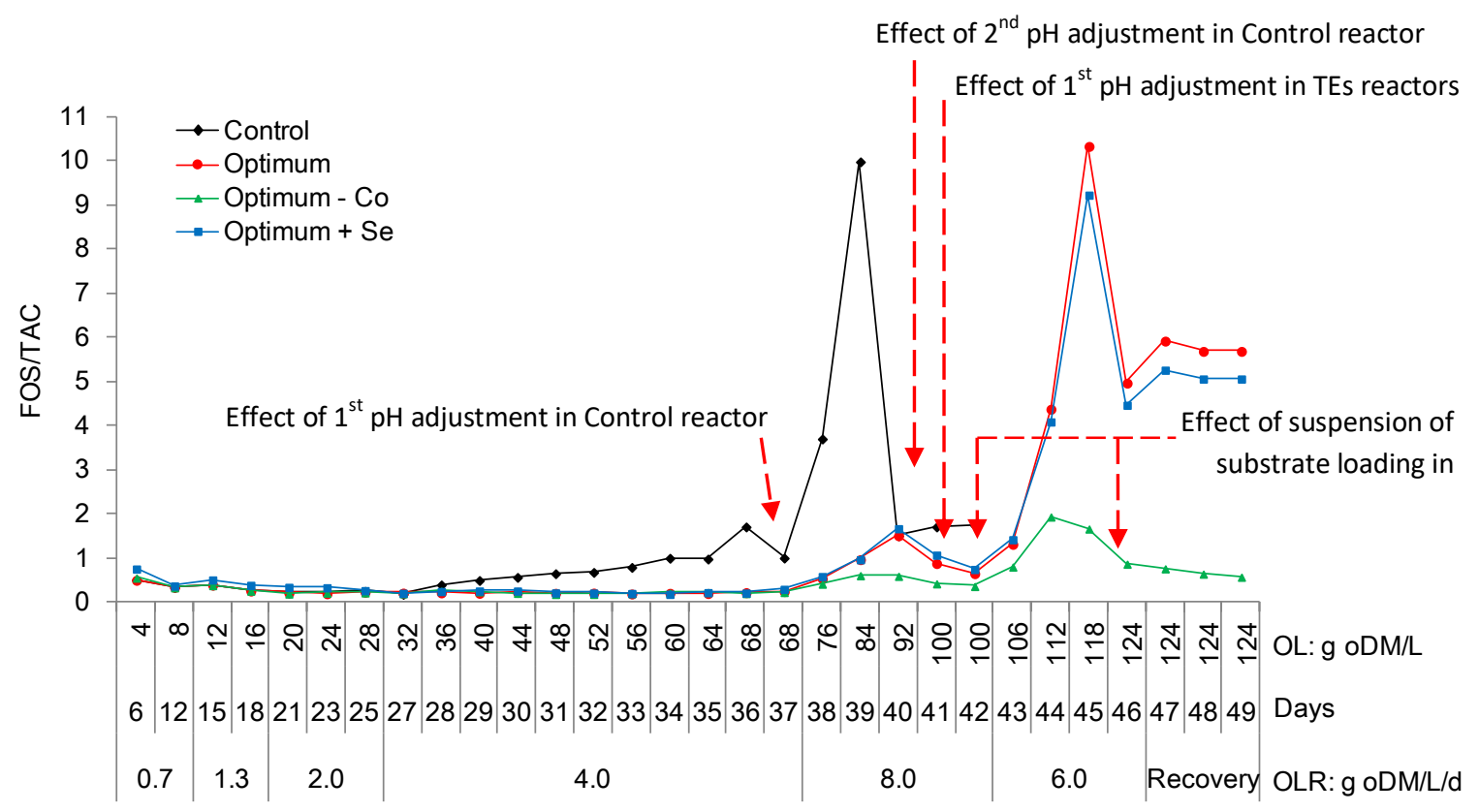

Figure 4 Overview of FOS/TAC of the reactors due to Ni, Co, Se and Mo supplementation and different OLR in the mesophilic semi-continuous experiment with mixed fruit residue.

Note: FOS/TAC values between $0.15-0.45$ indicate stability in the biomethanization process; and FOS/TAC > 0.6 indicate instability (Voss et al., 2009; Hach Lange $\mathrm{GmBH}, 2014$ ). 
Process Stability at OLR of $0.7 \mathrm{~g}$ to $2.0 \mathrm{~g}$ oDM/L/d: When OLR was increased from $0.7 \mathrm{~g}$ to $1.3 \mathrm{~g}$ oDM/L/d and maintained for 6 days, the FOS/TAC value in the Optimum +Se increased from 0.37 to 0.51 and stabilized at 0.39 at the end of the $1.3 \mathrm{~g}$ oDM/L/d OLR (Figure 4, day 18). Conversely, within the same period, the FOS/TAC values in the Control, Optimum, and Optimum -Co reactors decreased to 0.26 from 0.3 that was measured at the end of $0.7 \mathrm{~g}$ $\mathrm{oDM} / \mathrm{L} / \mathrm{d}$ OLR. Increasing OLR from $1.3 \mathrm{~g}$ $\mathrm{oDM} / \mathrm{L} / \mathrm{d}$ to $2 \mathrm{~g}$ oDM/L/d and maintaining the OLR for 7 days did not increase FOS/TAC above 0.3 in any of the reactor, including the Optimum + Se reactor.

Process Stability at $\mathbf{4 g}$ ODM/L/d OLR: Figure 4 shows the FOS/TAC trend at $4 \mathrm{~g}$ $\mathrm{oDM} / \mathrm{L} / \mathrm{d}$ OLR in the four reactors. In the Control reactor, it was 0.18 in the start of the OLR of $4 \mathrm{~g}$ $\mathrm{oDM} / \mathrm{L} / \mathrm{d}$, but rose steadily to 1.71 at the end of this OLR when the total organic load was $68 \mathrm{~g}$ oDM/L (day 37). This necessitated the first suspension of substrate loading followed by $\mathrm{pH}$ adjustment to 7.0 in the Control reactor. This intervention caused the FOS/TAC to decrease to 0.98 . On the contrary, FOS/TAC value was less than 0.30 at all times of measurement in the Optimum, Optimum -Co and Optimum +Se reactors and remained so until the end of the OLR of $4 \mathrm{~g}$ oDM/L/d.

Process Stability at $8 g$ ODM/L/d OLR: Figure 4 shows FOS/TAC trend in the Control and its Variants. The Optimum -Co Variant had a peak FOS/TAC of 0.61 within the $8 \mathrm{~g}$ oDM/L/d OLR, whereas the Optimum and Optimum $+\mathrm{Se}$ reactors had peak FOS/TAC values of 1.51 and 1.67 respectively. Due to the high FOS/TAC values in the Optimum and the Optimum +Se reactors, all the three reactors were operated one day without substrate loading (after loading of $100 \mathrm{~g} \mathrm{oDM} / \mathrm{L}$ ) in order to degrade accumulated organic load. The one-day break in organic loading induced recovery and returned the FOS/TAC values to 0.35 (Optimum -Co), 0.65 (Optimum) and 0.75 (Optimum +Se). Conversely, the Control reactor had a peak FOS/TAC of 9.99 at OLR of $8 \mathrm{~g}$ oDM/L/d and this was accompanied by $\mathrm{pH}$ of 6.2. Adjustment of $\mathrm{pH}$ was initiated and resulted in the decrease in FOS/TAC value of the Control reactor from 9.99 to 1.51 . Substrate loading was stopped in the Control reactor at a total organic loading of $84 \mathrm{~g}$ $\mathrm{oDM} / \mathrm{L}$ and recovery was monitored. Notwithstanding these interventions, the FOS/TAC in the Control reactor varied between 1.51 and 1.93 until the end of the study (day 49).

To evaluate the ability of the TEs configurations to restore normal biomethanization in the acidified digesters, the instability-inducing $8 \mathrm{~g}$ oDM/L/d OLR was suspended and $6 \mathrm{~g}$ oDM/L/d OLR was maintained until day 46 in the Optimum, Optimum -Co and Optimum +Se reactors. The FOS/TAC and $\mathrm{pH}$ values associated with the $6 \mathrm{~g} \mathrm{oDM} / \mathrm{L} / \mathrm{d}$ OLR are shown in Figure $5 a-c$. 

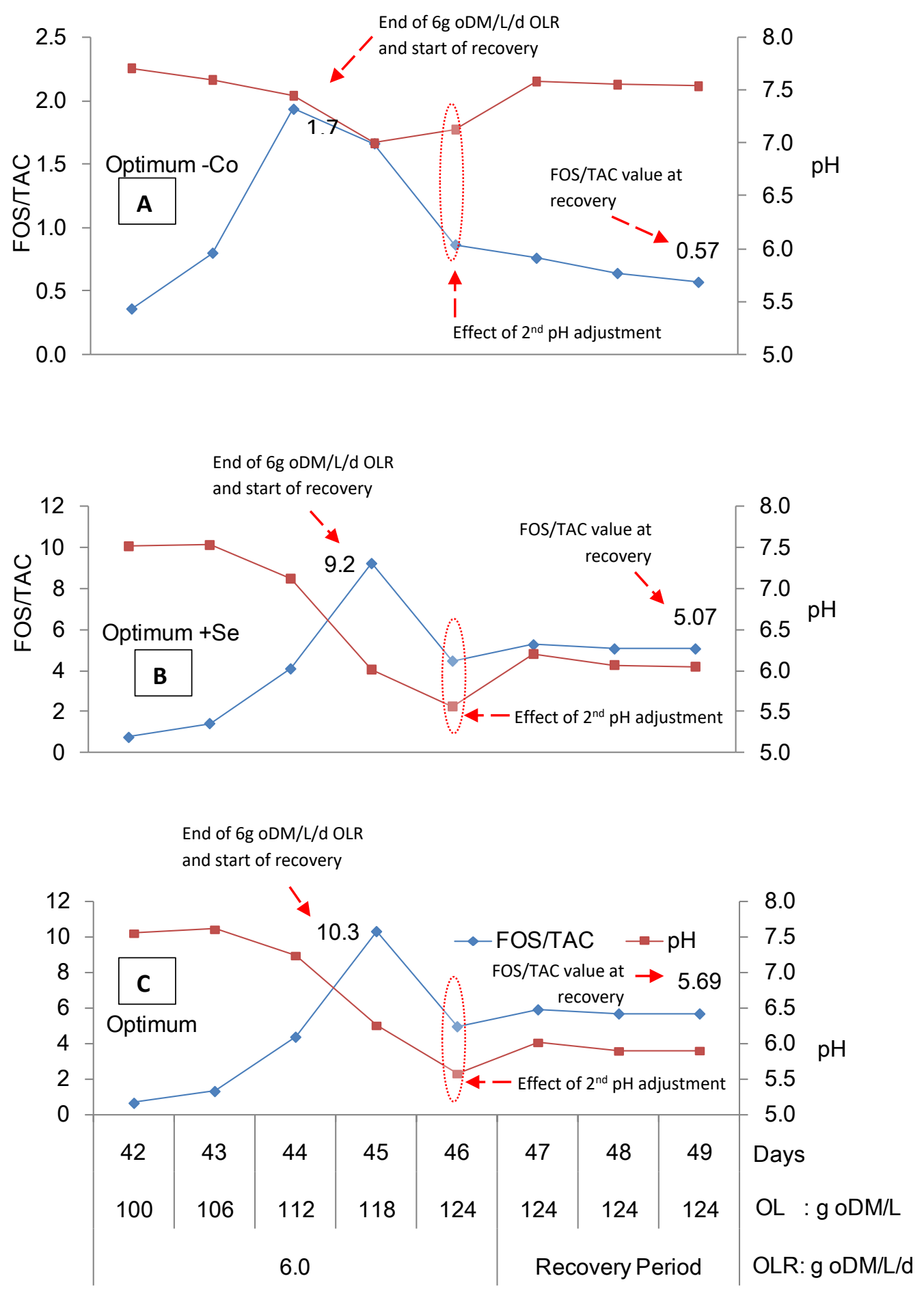

Figure 5a-c FOS/TAC of the (A) Optimum - $\mathrm{Co}$, (B) Optimum +Se and (C) Optimum reactors due to $\mathrm{Ni}, \mathrm{Co}$, Se and Mo supplementation and OLR of $6 \mathrm{~g}$ oDM/L/d in the mesophilic semi-continuous experiment fed with mixed fruit residue. (TEs configurations of the Optimum, Optimum +Se and Optimum -Co reactors are shown in Table 1) 
Peak FOS/TAC of 9.2 and 10.3 were recorded in the Optimum $+\mathrm{Se}$ and the Optimum reactors respectively after substrate loading resumed at $6 \mathrm{~g} \mathrm{oDM/L/d} \mathrm{OLR.} \mathrm{Peak} \mathrm{FOS/TAC} \mathrm{value} \mathrm{of} 1.66$ was also recorded in the Optimum -Co within the same phase of OLR. The sharp rise in FOS/TAC was accompanied by noticeable decreases in $\mathrm{pH}$ values in Optimum $+\mathrm{Se}$ and Optimum reactors, similar to the Control treatment at failure point. The corresponding $\mathrm{pH}$ in the reactors at peak FOS/TAC were 6.26 (Optimum), 6.02 (Optimum $+\mathrm{Se}$ ) and 7.0 (Optimum -Co). Consequently, substrate loading was stopped and $\mathrm{pH}$ was adjusted to 7.0 with $1 \mathrm{M} \mathrm{NaOH}$ in the Optimum +Se and Optimum reactors. Notwithstanding the $\mathrm{pH}$ adjustment after organic loading was stopped (day 46), FOS/TAC in the Optimum +Se and Optimum reactors stayed at 5.07 and 5.69 respectively at the end of the study. Conversely, the FOS/TAC value dropped from 1.66 to 0.57 in the Optimum -Co reactor on day 49.

Individual VFAs Fingerprints of the Reactors: The plots of acetic acid, propionic acid, n-butyric acid, iso-butyric acid, n-valeric acid and iso-valeric acid (VFAs concentration) against Days are shown in Figure 6a-d for Optimum, Control, Optimum +Se and Optimum -Co reactors from day $12(0.7 \mathrm{~g}$ oDM/L/d) to 49 (recovery period). More pertinent due to associated instability are VFAs fingerprints during the $8 \mathrm{~g}$ - and $6 \mathrm{~g}$ oDM/L/d. The VFAs concentrations were mainly due to accumulations of acetic acid, propionic acid and butyric acid.

In the Control reactor, acetic acid concentration $(\mathrm{g} / \mathrm{L})$ was between $4.2 \pm 0.2$ and $5.2 \pm 0.7$ during the OLR of $8 \mathrm{~g} \mathrm{oDM} / \mathrm{L} / \mathrm{d}$ (days 38 and 42 ). During the same OLR and days, acetic acid concentrations $(\mathrm{g} / \mathrm{L})$ were between $0.5 \pm 0.05$ and $2.0 \pm 0.03$ in the Optimum; $0.5 \pm 0.04$ and $2.5 \pm 0.9$ in Optimum $+\mathrm{Se}$; and $0.6 \pm 0.07$ and $0.2 \pm 0.02$ in the Optimum -Co reactors. Peak propionic acid and butyric acid concentrations $(\mathrm{g} / \mathrm{L})$ during the OLR of $8 \mathrm{~g}$ oDM/L/d were as follows: Control (1.2 for butyric only); Optimum $(0.2 \pm 0.03$ and $0.09 \pm 0.003$ respectively), Optimum +Se (0.4 and 0.9 respectively), Optimum -Co $(0.2 \pm 0.02$ and $0.06 \pm 0.01$ respectively).

During the OLR of $6 \mathrm{~g} o D M / L / d$, the individual VFAs had accumulated from the OLR of $8 \mathrm{~g}$ $\mathrm{oDM} / \mathrm{L} / \mathrm{d}$ so that in the Optimum reactor, the individual VFAs concentrations $(\mathrm{g} / \mathrm{L})$ on day 49 were acetic acid, $6.3 \pm 1$; propionic acid, $0.25 \pm 0.02$; and butyric acid, $0.7 \pm 0.05$. In the Optimum -Co reactor the VFAs concentrations $(\mathrm{g} / \mathrm{L})$ were acetic acid, $0.6 \pm 0$; propionic acid $0.7 \pm 0.04$ and butyric acid, $0.6 \pm 0.05$. In the Optimum + Se reactor, these were $(\mathrm{g} / \mathrm{L})$, acetic acid, 6.1 \pm 0.09 ; propionic acid, $0.3 \pm 0.03$; and butyric acid, $0.8 \pm 0.02$. The Control reactor was not operational in this phase. 
Ezebuiro and Körner / Nig. J. Biotech. Vol. 38 Num. 1: 179-193 (June 2021)
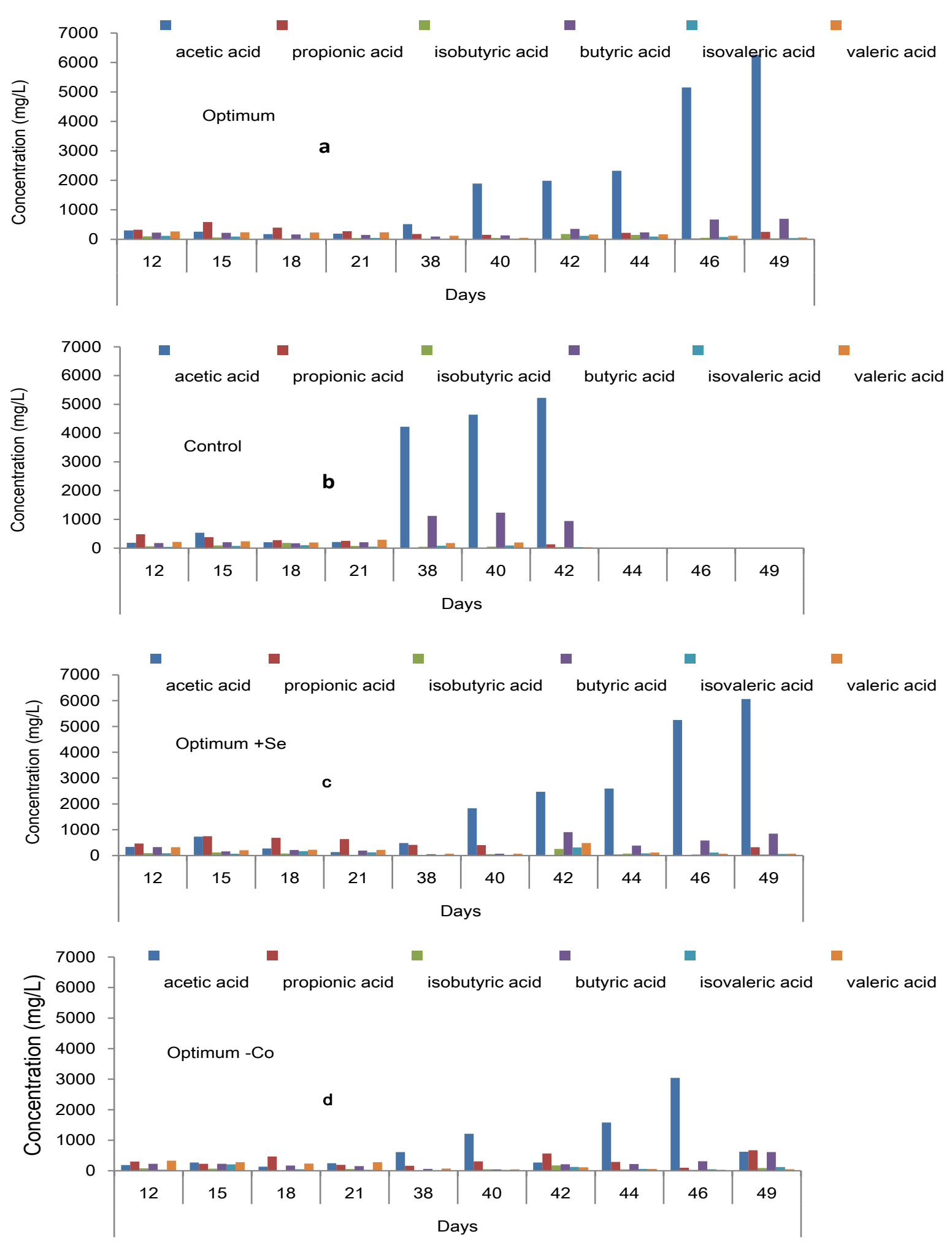

Figure 6a-d Individual VFAs fingerprints in the experimental reactors due to OTC and its Variants during the mesophilic semi-continuous experiment with mixed fruit residue: a) Optimum reactor; b) Control reactor; c) Optimum +Se reactor; d) Optimum -Co reactor 
Although the Optimum -Co reactor had the lowest acetic acid and butyric acid accumulation compared to the Optimum and Optimum +Se reactors, it had a lower propionic acid oxidation capability compared to the other reactors. This is highlighted in Figure 6e for days 40 to 49 during the $8 g-, 6 g$ oDM/L/d and recovery period. Within this period, the peak propionic acid concentrations $(\mathrm{g} / \mathrm{L})$ of the reactors include: Optimum, 0.3; Optimum $+\mathrm{Se}, \quad 0.4 ;$ and Optimum -Co, 0.7 .

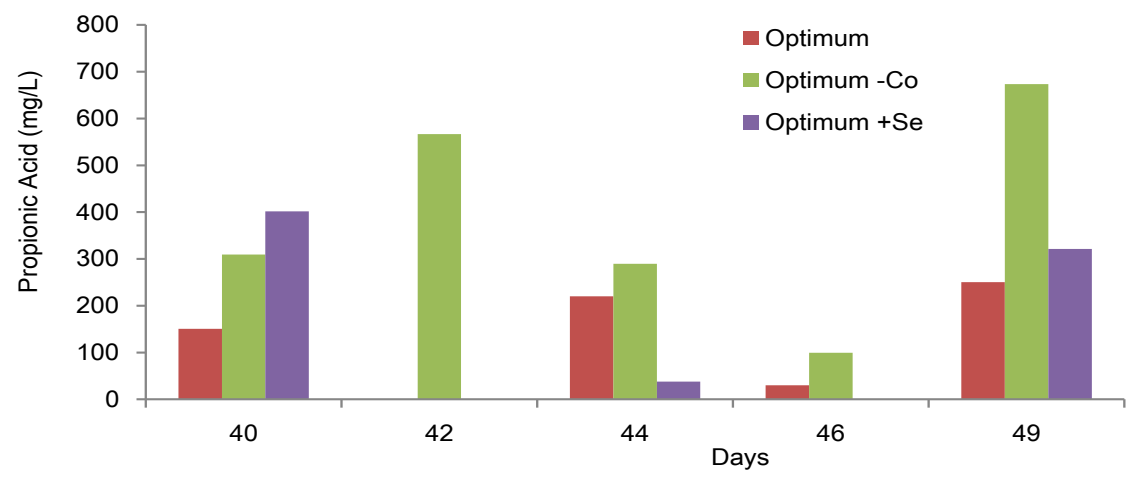

Figure 6e Propionic acid fingerprint during mesophilic biomethanization of mixed fruit residue in the Optimum, Optimum -Co and Optimum +Se reactors between days 40 and 49

\section{Discussion}

The results show potentials for maintaining process stability during high organic loading by manipulating and adapting previously identified VFAs*TEs. The observed biochemical behaviors of Optimum, Optimum -Co and Optimum +Se formulations could be explained in relation to the VFAs*Co and VFAs*Se. The biochemical behavior of VFAs*Se in this study is consistent with the RSM in Figure 1 and shows that increase in Se concentration in the Optimum + Se reactor (relative to Se Conc. in the Optimum) improved $\mathrm{CH}_{4}$ production and FOS/TAC relative to the Optimum. The adaptation or lag period of $18-25$ days prior to the observation of positive effect of the VFAs*Se is consistent with earlier findings on adaptation to TEs toxicity during methanization (Ezebuiro and Koerner, 2017).

Antagonistic pre-adaptation effects of the VFAs*Se at the low OLRs (Table 2) as seen in Optimum +Se Variant of OTC is also consistent with the RSM in Figure 1 that suggests that relative to an OTC, Se concentration should decrease at low OLR and vice versa. The Se concentration in the Optimum + Se reactor was $1.5 \mathrm{mg} / \mathrm{L}$, which is higher than $0.40 \mathrm{mg} / \mathrm{L}$ that is Optimum for $120 \mathrm{mmol} / \mathrm{L}$ VFAs or medium OLR. Hence, lower OLR such as $0.7 \mathrm{~g}-2.0 \mathrm{~g}$ oDM/L/d require $\mathrm{Se}$ concentration $<1.5 \mathrm{mg} / \mathrm{L}$. Supposedly, the inhibitory high Se concentration of the Optimum +Se Variant $(1.5 \mathrm{mg} / \mathrm{L}$, compared to $0.4 \mathrm{mg} / \mathrm{L}$ in Optimum and Optimum -Co) was antagonistic and accounted for the observed poorer $\mathrm{CH}_{4}$ production and lower process stability in the low OLR phase compared to the Optimum and the Optimum Co reactors. From Table 1, $0.40 \mathrm{mg} / \mathrm{L} \mathrm{Se}$, as in Optimum and Optimum -Co, is most appropriate for medium VFAs level of $120 \mathrm{mmol} / \mathrm{L}$ (or medium OLR in Table 2). However, Optimum $+\mathrm{Se}$ Variant showed better $\mathrm{CH}_{4}$ production (Figure $3 a-c$ ) and process stability (Figure $5 a-c$ ) than the Optimum at OLR $\geq 4.0 \mathrm{~g}$ oDM/L/d. This is consistent with RSM in Figure 1 that suggests that a higher Se concentration relative to Se concentration in the OTC is required as VFAs concentration or OLR increases.

Similarly, from Figure 1, Co has an inverse and negative relationship with VFAs. The VFA*Co in Figure 1 was adapted to high OLR by lowering Co concentration from $2.7 \mathrm{mg} / \mathrm{L}$ in the Optimum, to $0.5 \mathrm{mg} / \mathrm{L}$ in the Optimum -Co. This was in anticipation of a corresponding positive biochemical effect during a high OLR. The adapted VFA*Co in the Optimum -Co Variant was responsible for the superior $\mathrm{CH}_{4}$ production and FOS/TAC, especially at $6 \mathrm{~g} \mathrm{oDM} / \mathrm{L} / \mathrm{d}$ and the recovery period when FOS/TAC remained within the stability range in spite of the high OLR regime. Figure $6 \mathrm{~d}$ and $6 \mathrm{e}$ showed that although the Optimum -Co Variant had a higher potential 
for $\mathrm{CH}_{4}$ production, process stability and recovery from acidification, it had weak propionic acid degradation ability. This observation is consistent with earlier publications on the role of $\mathrm{Co}$ in propionic acid degradation (Halarnkar and Blomquist, 1989; Ezebuiro et al., 2018). Hence, lower propionic acid oxidation potential as was evident in the Optimum -Co reactor (Co, $0.5 \mathrm{mg} / \mathrm{L})$ compared to Optimum and the Optimum + Se reactors (2.7 $\mathrm{mg} / \mathrm{L}$ Co each) was anticipated. At OLR of $4.0 \mathrm{~g}$ $\mathrm{oDM} / \mathrm{L} / \mathrm{d}$, the OTC outperformed its variants on $\mathrm{CH}_{4}$ production and process stability. This is consistent with the RSM model in Figure 1 that produced the OTC in Table 1, which was designed to be most efficient for medium OLR $(120 \mathrm{mmol} / \mathrm{L})$ for all methanization processes.

\section{Acknowledgement}

The authors are thankful to TUHH for funding the laboratory analyses. The laboratories of the Institute for Wastewater Management and Water Protection, and the Institute for Environmental Technology and Energy Economics at the TUHH are strongly appreciated for providing special analytical support. Prof. AnPing Zeng is highly appreciated for cosupervising the study. Finally, the authors are grateful to the National Biotechnology Development Agency for releasing author ${ }^{\mathrm{a}}$ for the study.

\section{Funding}

The scholarship enabling author ${ }^{\mathrm{a}}$ to conduct this study at the Hamburg University of Technology (TUHH), Germany, was granted by the German Academic Exchange Programme (DAAD): grant number $\mathrm{A} / 10 / 90006$.

\section{References}

Alonso, E., Aparicio, I., Santos, J. L., Villar, P. and Santos, A. (2009). Sequential extraction of metals from mixed and digested sludge from aerobic WWTPs sited in the south of Spain. Waste manag. 29(1), 418-24. Doi: 10.1016/j.wasman.2008.01.009.

Amani, T., Nosrati, M., Mousavi, S. M. and Kermanshahi, R. K. (2010). Study of syntrophic anaerobic digestion of volatile fatty acids using enriched cultures at mesophilic conditions. Int. J. Environ. Sci. Technol. 8(1), 83-96. Doi: 10.1007/BF03326198.
Ariunbaatar, J., Esposito, G., Yeh, D. and Lens, P. (2016). Enhanced anaerobic digestion of food waste by supplementing trace elements: Role of selenium (vi) and iron (ii). Front. Environ. Sci. 4(8), 1-11. https://doi.org/DOI $10.3389 /$ fenvs. 2016.00008

Bardi, M. and Aminirad, H. (2020). Synergistic effects of co-trace elements on anaerobic codigestion of food waste and sewage sludge at high organic load. Environ. Sci. Pollut. Res. 27, 18129-18144. doi: DOI 10.1007/s11356-02008252-y.

Braga, A. F. M., Pereira, M. B., Zaiat, M., Gustavo H., Da Silva, R. and Fermoso, F. G. (2018). Screening of trace metal supplementation for black water anaerobic digestion. Environ. Technol. 39(14), 1776-1785, DOI: $10.1080 / 09593330.2017 .1340343$

Da Silva, J. A., Braga, A. F. M., Fermoso, F. G., Zaiat. M and Gustavo H. R. S. (2021). Evaluation of the influence of trace metals on methane production from domestic sewage, using the Plackett-Burman experimental design. J. Environ. Manage. 294, 113002. DOI: 10.1016/j.jenvman.2021.113002

Ezebuiro, N. C. and Koerner, I. (2017). Characterization of anaerobic digestion substrates regarding trace elements and determination of the influence of trace elements on the hydrolysis and acidification phases during the methanization of a maize silage-based feedstock. J. Environ. Chem. Eng. 5(1), 341351. Doi: 10.1016/j.jece.2016.11.032.

Ezebuiro, N. C., Techamanoon, K. and Koerner, I. (2018). Synergistic and antagonistic influences of trace elements on volatile fatty acids degradation and methane production during the methanization of a mixture of volatile fatty acids. J. Environ. Chem. Eng. 6(1), 1455-1467. Doi: https://doi.org/10.1016/j.jece.2017.12.017.

Facchin, V., Cavinato, C., Pavan, P. and Bolzonella, D. (2013). Batch and continuous mesophilic anaerobic digestion of food waste: Effect of trace elements supplementation. Chem. Eng. Trans. 32, 457-462. Doi: DOI 10.3303/CET1332077. 
Halarnkar, P. P. and Blomquist, G. J. (1989). Comparative aspects of propionate metabolism. Comp. Biochem. Physiol. 92(2), 227-31. Available at: http://www.ncbi.nlm.nih.gov/pubmed/2647392.

Pobeheim, H., Munk, B., Lindorfer, $H$. and Guebitz, G. M. (2011). Impact of nickel and cobalt on biogas production and process stability during semi-continuous anaerobic fermentation of a model substrate for maize silage. Water Res. 45, 781-787. https://doi.org/10.1016/j.watres.2010.09.001

Maharaj, B. C., Mattei, M. R., Frunzo, L., Hullebusch, D. Y. and Esposito, G. (2018). ADM1 based mathematical model of trace element complexation in anaerobic digestion processes. Bioresour. Technol. 276, 253-259

Mancini, G., Papirio, S., Riccardelli, G, Lens, P. N. L. and Esposito, G. (2018). Trace elements dosing and alkaline pretreatment in the anaerobic digestion of rice straw. Bioresour. Technol. 247, 897-903.

DOI: $10.1016 /$ j.biortech.2017.10.001

Myszograj, S., Stadnik, A. and PłuciennikKoropczuk, E. (2018). The Influence of Trace Elements on Anaerobic Digestion Process. Civ. Environ. Eng. Rep. 28(4), 105-115. DOI: 10.2478/ceer-2018-0054

Qiang, H., Niu, Q., Chi, Y. and Li, Y. (2013). Trace metals requirements for continuous thermophilic methane fermentation of high-solid food waste. Chem. Eng. J. 222, 330-336. Doi: DOI 10.1016/j.cej.2013.02.076.

Suich, R. and Derringer, G. (1980). Simultaneous optimization of several response variables. J. Qual. Technol. 12(4), 214-219.

Tokalioğlu, Ş. , Kartal, Ş., and Birol, G. (2003). Application of a three-stage sequential extraction procedure for the determination of extractable metal contents in highway soils. Turkish J. Chem. 27(3), 333-346.

Ünal, B., Perry, V. R., Sheth, M., Gomez-Alvarez, V., Chin, K. J. and Nüsslein, K. (2012). Trace elements affect methanogenic activity and diversity in enrichments from subsurface coal bed produced water. Front. Microbiol. 3, 1-14. https://doi.org/10.3389/fmicb.2012.00175

Verein Deutscher Ingenieure (2006). 4630 Fermentation of organic materials, characterisation of the substrate, sampling, collection of material data, fermentation tests. Germany.

Voss, E., Weichgrebe, D. and Rosenwinkel, K. H. (2009). FOS/TAC-Deduction, Methods, Application and Significance, in Internationale Wissenschaftskonferenz "Biogas Science 2009 science meets practice". Erding: LfL-Bayern.

Yazdanpanah, A., Ghasimi, D., Kim, M., Nakhla, G. and Hafez, H. (2018). Impact of trace element supplementation on mesophilic anaerobic digestion of food waste using Fe-rich inoculum. Environ. Sci. Pollut. Res. 25(29), 29240-29255. Doi:10.1007/s11356-018-2832-2.

Zhang, Y., Walker, M. and Banks, C. (2010). Technical Report: Optimizing Processes for the Stable Operation of Food Waste. Defra project code WR1208, Pp $17-40$. 